\title{
Pênfigo vulgar: uma manifestação cutânea autoimune na síndrome pós-Covid-19
}

\author{
Rebecca Castelo Branco (D) Antônio Urbano Monteiro-Neto (iD) Vanessa Lucilia Silveira de \\ Medeiros
}

Universidade Federal de Pernambuco, Recife, Pernambuco, Brazil

\section{$\triangle$}

Vanessa Lucília Silveira de Medeiros Clínica de Dermatologia, Hospital das Clínicas da Universidade Federal de Pernambuco, Av. Prof. Moraes Rego - 1235. CEP: 50670-901, Cidade Universitária, Recife-PE

vanessalucilia@yahoo.com.br

Editado por:

Fernando Cruz
A relação entre autoimunidade e doenças virais vem se confirmando progressivamente. Sabe-se que inúmeros vírus podem estar associados ao desenvolvimento de doenças inflamatórias crônicas e autoimunes', a exemplo do Parvovírus B 192, vírus Epstein-Barr $(E B V)^{3}$, Citomegalovírus (CMV), vírus da hepatite $A$ e $C 4$ vírus Herpes-6, HTLV-1 e o vírus da rubéola. ${ }^{4 \cdot 6}$

O coronavírus da síndrome respiratória aguda grave 2 (SARS-CoV-2) vem demonstrando ser um gatilho para a autoimunidade desde o início da pandemia. Os relatos de doenças como a Síndrome de Guillain-Barré7,8, síndrome de Miler Fisher', síndrome do anticorpo antifosfolípide $^{10}$, púrpura trombocitopênica imune (PTI) ${ }^{11}$, lúpus eritematoso sistêmico ${ }^{12} \mathrm{e}$ Doença de Kawasaki ${ }^{13}$ foram descritos em pacientes que apresentaram infecção por SARS-CoV-2, demonstrando seu efeito na mudança da expressão imunológica em indivíduos geneticamente predispostos.

Neste artigo publicado na SN Comprehensive Clinical Medicine ${ }^{14}$ relatamos um caso de pênfigo vulgar (PV) após infecção por SARS-CoV-2. O PV é uma doença autoimune bolhosa que ocorre devido a produção de anticorpos do tipo lgG 4, assim como a PTI ${ }^{15}$, contra a desmogleína 1 e 3 presentes na pele e mucosas. $\bigcirc$ fenômeno ocorre pela presença de um estímulo altamente imunogênico, que leva a produção de múltiplos anticorpos e posteriormente autoanticorpos no intuito de destruir o patógeno. Existiam relatos anteriores de PV após infecções por Varicela Zoster e Herpes zoster, agora também é demonstrado pelo SARS-CoV-2. Os pênfigos podem ser desencadeados temporalmente distante da infecção, assim como a doença de Kawasaki (13) o que dificulta fazer a correlação entre os fato. Por isso, deve-se estar atento e questionar ativamente antecedentes de infecções virais frente a um quadro recente de PV. 
Rebecca Castelo Branco

https://orcid.org/0000-0002-0804-9111

Antônio Urbano Monteiro-Neto

https://orcid.org/ 0000-0003-3305-848X

Vanessa Lucilia Silveira de Medeiros

https://orcid.org/ 0000-0002-1445-587X

\section{Referências}

1. Barzilai $O$, Ram M and Shoenfeld Y. Viral infection can induce the production of autoantibodies. Curr Opin Rheumatol 2007; 19(6):636-643 Doi:10.1097/BOR.0b013e$3282 f 0 a d 25$

2. Pavlovic M, Kats A, Cavallo M and Shoenfeld Y. Clinical and molecular evidence for association of SLE with parvovirus B19. Lupus 2010;19(7):783-792 Doi:10.1177/0961203310365715

3. Barzilai $O$, Sherer Y, Ram M, Izhaky D, Anaya JM and Shoenfeld $Y$. Epstein-Barr virus and cytomegalovirus in autoimmune diseases: are they truly notorious? A preliminary report. Ann N Y Acad Sci 2007; 1 108:567-577 Doi: $10.1196 /$ annals. 1422.059

4. Maya R, Gershwin ME and Shoenfeld Y. Hepatitis B virus (HBV) and autoimmune disease. Clin Rev Allergy Immunol 2008;34(1):85-102 Doi:10.1007/s12016-007-8013-6

5. Halpert $G$ and Shoenfeld Y. SARS-CoV-2, the autoimmune virus. Autoimmun Rev 2020;19(12): 102695 Doi:10.1016/i.autrev.2020.102695

6. Shoenfeld Y, Selmi C, Zimlichman E and Gershwin ME. The autoimmunologist: geoepidemiology, a new center of gravity, and prime time for autoimmunity. J Autoimmun 2008;31(4):325-330 Doi:10.1016/i.jaut.2008.08.004

7. Sedaghat Z and Karimi N. Guillain Barre syndrome associated with COVID-19 infection: A case report. J Clin Neurosci 2020;76:233-235 Doi:10.1016/i. jocn.2020.04.062

8. Toscano G, Palmerini F, Ravaglia S, Ruiz L, Invernizzi P, Cuzzoni MG, .. . Micieli G. Guillain-Barré Syndrome Associa- ted with SARS-CoV-2. N Engl J Med 2020;382(26):2574 2576 Doi:10.1056/NEJMc2009191

9. Manganotti P, Pesavento V, Buoite Stella A, Bonzi L, Campagnolo E, Bellavita $G$, . . . Luzzati R. Miller Fisher syndrome diagnosis and treatment in a patient with SARS-CoV-2. J Neurovirol 2020;26(4):605-606 Doi: 10. 1007/s 13365 020-00858-9

10. Zhang $Y$, Cao W, Jiang W, Xiao M, Li Y, Tang N, . . Zhu T. Profile of natural anticoagulant, coagulant factor and anti-phospholipid antibody in critically ill COVID-19 patients. J Thromb Thrombolysis 2020;50(3):580-586 Doi: 10.1007/s 1 1239-020-02182-9

11. Zulfiqar $A A$, Lorenzo-Villalba $N$, Hassler $P$ and Andrès $E$. Immune Thrombocytopenic Purpura in a Patient with Covid-19. N Engl J Med 2020;382(18):e43 Doi:10.1056/ NEJMc2010472

12. Bonometti R, Sacchi MC, Stobbione P, Lauritano EC, Tamiazzo S, Marchegiani A, . . Boverio R. The first case of systemic lupus erythematosus (SLE) triggered by COVID-19 infection. Eur Rev Med Pharmacol Sci 2020;24(18):96959697 Doi:10.26355/eurrev_202009_23060

13. Verdoni L, Mazza A, Gervasoni A, Martelli L, Ruggeri $M$, Ciuffreda $M, \ldots$ D'Antiga L. An outbreak of severe Kawasaki-like disease at the Italian epicentre of the SARS-CoV-2 epidemic: an observational cohort study. Lancet 2020;395(10239):1771-1778 Doi:10.1016/ s0140-6736(20)31 103-x

14. Medeiros VLS, Monteiro-Neto AU, França DDT, Castelo Branco R, Miranda Coelho É O and Takano DM. Pemphigus Vulgaris After COVID-19: a Case of Induced Autoimmunity. SN Compr Clin Med 2021: 1-5 Doi: 10.1007/ s42399-021-00971-8

15. Koneczny I, Yilmaz V, Lazaridis K, Tzartos J, Lenz TL, Tzartos S, . . . Leypoldt F. Common Denominators in the Immunobiology of IgG4 Autoimmune Diseases: What Do Glomerulonephritis, Pemphigus Vulgaris, Myasthenia Gravis, Thrombotic Thrombocytopenic Purpura and Autoimmune Encephalitis Have in Common? Front Immunol 2020; 11 :605214 Doi:10.3389/fimmu.2020.605214 